\title{
Modelling Cationic Diffusion in Nickel-Based Honeycomb Layered Tellurates using Vashishta-Rahman Interatomic Potential and Relevant Insights
}

\author{
Kartik Sau, ${ }^{1, *}$ Tamio Ikeshoji ${ }^{1}$ Godwill Mbiti Kanyolo, ${ }^{2}$ and Titus Masese ${ }^{3, \dagger}$ \\ ${ }^{1}$ Mathematics for Advanced Materials Open Innovation Laboratory (MathAM-OIL), \\ National Institute of Advanced Industrial Science and Technology (AIST), \\ c/o Advanced Institute for Materials Research (AIMR), Tohoku University, Sendai 980-857r, JAPAN \\ ${ }^{2}$ The University of Electro-Communications, Department of Engineering Science, 1-5-1 Chofugaoka, Chofu, Tokyo 182-8585 \\ ${ }^{3}$ Research Institute of Electrochemical Energy (RIECEN), \\ National Institute of Advanced Industrial Science and Technology (AIST), \\ 1-8-31 Midorigaoka, Ikeda, Osaka 563-8577, Japan
}

\begin{abstract}
Although the fascinatingly rich crystal chemistry of honeycomb layered oxides has been accredited as the propelling force behind their remarkable electrochemistry, the atomistic mechanisms surrounding their operations remain unexplored. Thus, herein, we present an extensive molecular dynamics study performed systematically using a reliable set of inter-atomic potential parameters of $A_{2} \mathrm{Ni}_{2} \mathrm{TeO}_{6}$ (where $A=\mathrm{Li}, \mathrm{Na}$, and $\mathrm{K}$ ). We demonstrate the effectiveness of the Vashishta-Rahman form of the inter-atomic potential in reproducing various structural and transport properties of this promising class of materials and predict an exponential increase in cationic diffusion with larger inter-layer distances. The simulations demonstrate the correlation between broadened inter-layer (inter-slab) distances associated with the larger ionic radii of $\mathrm{K}$ and $\mathrm{Na}$ compared to $\mathrm{Li}$ and the enhanced cationic conduction exhibited in $\mathrm{K}_{2} \mathrm{Ni}_{2} \mathrm{TeO}_{6}$ and $\mathrm{Na}_{2} \mathrm{Ni}_{2} \mathrm{TeO}_{6}$ relative to $\mathrm{Li}_{2} \mathrm{Ni}_{2} \mathrm{TeO}_{6}$. Whence, our findings connect lower potential energy barriers, favourable cationic paths and wider bottleneck size along the cationic diffusion channel within frameworks (comprised of larger mobile cations) to the improved cationic diffusion experimentally observed in honeycomb layered oxides. Furthermore, we elucidate the role of inter-layer distance and cationic size in cationic diffusion. Our theoretical studies reveal the dominance of inter-layer distance over cationic size, a crucial insight into the further performance enhancement of honeycomb layered oxides.
\end{abstract}

\section{INTRODUCTION}

As the winds of change continue to push humanity to the crescendo of an energy revolution, high-energydensity storage systems have gained momentous traction, with lithium-ion batteries at the epicentre of both commercial and industrial applications.[1-3] This dominance of lithium-ion chemistries is principally propelled by their low redox potential (-3.04 V versus standard hydrogen electrode (SHE)) along with expedient ion kinetics that facilitate the development of layered oxide cathode materials that engender batteries with high voltages, highpower-densities and excellent cyclability. [2, 3] However, the sustainability of lithium-ion batteries is heavily impeded by the prohibitive costs, safety issues and scarcity of lithium resources, galvanising explorations into alternative chemistries with terrestrial affluence such as sodium-ion and potassium-ion batteries. $[4,5]$ Although the low redox potentials of $\mathrm{Na}$ and $\mathrm{K}(-2.71 \mathrm{~V}$ and -2.93 $\mathrm{V}$ versus SHE in aqueous electrolytes, respectively) earmark their potential as Li-ion alternatives, their layered oxide analogues have been found to yield low average voltages $(\sim 3 \mathrm{~V})$ due to their large ionic sizes. [6]

In an effort to ameliorate the electrochemical performance and structural stability of layered oxides, partial substitution of the transition metal species with other

\footnotetext{
* kartik.sau@gmail.com

$\dagger$ titus.masese@aist.go.jp
}

transition metal species or highly valent species (such as chalcogens or pnictogens) has been employed. In this pursuit, a unique class of heterostructures known as honeycomb layered oxides has garnered prominence as high-energy-density cathode materials for their exquisite crystal structures that engender remarkable ion mobility and high voltages even with $\mathrm{Na}$ and K chemistries.[7-19] These materials typically adopt chemical compositions of $A_{2} M_{2} D \mathrm{O}_{6}, A_{3} M_{2} D \mathrm{O}_{6}$ or $A_{4} M D \mathrm{O}_{6}$ (where $M$ can be divalent or trivalent transition or coinage-metal atoms such as $\mathrm{Cr}, \mathrm{Mn}, \mathrm{Fe}, \mathrm{Co}, \mathrm{Ni}, \mathrm{Cu}$ or some combination thereof; $D$ represents pentavalent or hexavalent metal atoms such as $\mathrm{Sb}, \mathrm{Te}, \mathrm{Bi}$; and $A$ can be alkali atoms such as $\mathrm{Li}, \mathrm{Na}$, $\mathrm{K}$, etc. $[7,20]$ Given the differences in their ionic sizes and valency states, the 2:1 atomic ratio of $M$ and $D$ (such as in $A_{2} M_{2} D \mathrm{O}_{6}$ and $A_{3} M_{2} D \mathrm{O}_{6}$ ), renders an array of parallel transition metal slabs with a distinct honeycomb arrangement of multiple $M$ atoms surrounding $D$ atoms in a layered framework of interposed $A$ alkali atoms.

Fast ion kinetics and high voltage capabilities are key prerequisites of any energy material geared towards future capacious battery applications. In this quest, the fine-tuning of the honeycomb layered structures which involves the judicious selection of resident species has been the focal point of numerous studies on this subject.[21-26] For high capacity, trivalent or divalent metals with high structural stability and high redox voltages (such as $\mathrm{Ni}^{2+} / \mathrm{Ni}^{3+} / \mathrm{Ni}^{4+}$ in $A_{2} \mathrm{Ni}_{2} D \mathrm{O}_{6}, A_{3} \mathrm{Ni}_{2} D \mathrm{O}_{6}$ or $\left.A_{4} \mathrm{Ni} D \mathrm{O}_{6}\right)$ are favoured as they allow partial alkali atom occupation without disintegrating. [8, 10, 12-19, 27] 
For high voltages, highly valent pnictogen or chalcogen atoms such as $\mathrm{Te}, \mathrm{Bi}$ and $\mathrm{Sb}$ are employed to lower the covalency of the bond between the oxygen atoms and (di or tri) valent transition metals thereby increasing the energy required for (di or tri) valent cation oxidation. In turn, this induces a large increase in the overall voltage of the battery which can be predicted by the Pauling scale $(\mathrm{Te}>\mathrm{Sb}>\mathrm{Bi}$ ) of electronegativity. Indeed, tellurates used alongside $\mathrm{Ni}^{2+}$ in honeycomb layered oxides have been reported to produce the highest voltages (over 4V) to date.[8, 10, 12, 13, 15-18] Thus, for a comparative study on honeycomb layered oxide electrochemical performance, $\mathrm{Ni}^{2+}$-based honeycomb tellurates $\left(A_{2} \mathrm{Ni}_{2} \mathrm{TeO}_{6}\right)$ present an ideal pedagogical platform as a leverage for insights into the optimisation of related honeycomb layered oxides.

To surmise the mechanisms of honeycomb layered frameworks, a crystal structural illustration of $A_{2} \mathrm{Ni}_{2} \mathrm{TeO}_{6}(A=\mathrm{Li}, \mathrm{Na}$ and $\mathrm{K})$ is furnished in Figure 1. As shown, the $A$ atoms are interspersed between metal slabs consisting of $\mathrm{Ni}$ and $\mathrm{Te}$ in an octahedral coordination with oxygen atoms. The inter-slab (inter-layer) distances increase in the sequence of $\mathrm{Li}<\mathrm{Na}<\mathrm{K}$ in accord with the Shannon-Prewitt ionic size of the alkali atoms (Note that an ordered $\mathrm{Li}_{2} \mathrm{Ni}_{2} \mathrm{TeO}_{6}$ polymorph[12] is used for ease of comparison with the $\mathrm{Na}$ and $\mathrm{K}$ counterparts).[15, 21] As affirmed by previous structural studies on honeycomb layered frameworks, cations with smaller ionic radii (such as $\mathrm{Li}^{+}$in $\mathrm{Li}_{2} \mathrm{Ni}_{2} \mathrm{TeO}_{6}$ ) are characterised by alkali planes with smaller inter-layer distances (consequently stronger inter-layer bonds) which require more energy to facilitate two-dimensional (2D) cation diffusion during (de)insertion processes. [7] On the other hand, resident cations with larger ionic radii (such as $\mathrm{Na}^{+}$and $\mathrm{K}^{+}$in $\mathrm{Na}_{2} \mathrm{Ni}_{2} \mathrm{TeO}_{6}$ and $\mathrm{K}_{2} \mathrm{Ni}_{2} \mathrm{TeO}_{6}$ ) foster weaker inter-layer bonds that facilitate facile $2 \mathrm{D}$ cationic diffusion during battery operations As such, Ni-based honeycomb tellurate compositions comprising $\mathrm{Na}$ and $\mathrm{K}$ have demonstrated faster cationic mobilities relative to their Li-based counterparts, with $\mathrm{K}$ displaying the lowest energy barrier for cationic mobility (viz., activation energy).[28, 29]

Despite the immense potential envisioned in the experimental and theoretical explorations of honeycomb layered materials, details surrounding the various mesoscopic mechanisms engendering their remarkable electrochemical performance still remain unexplored. Specifically, bigger size cations show higher activation energy barrier, resulting in lower cationic diffusion. This series of materials, $A_{2} \mathrm{Ni}_{2} \mathrm{TeO}_{6}(A=\mathrm{Li}, \mathrm{Na}$ and $\mathrm{K})$, show completely contrasting behaviour. Thus, it attracts theoretical attention to understand the origin of such contrasting behaviour. In light of this, computational methodologies such as molecular dynamics (MD) simulations present a unique avenue to unveil the microscopic physicochemical properties such as ion transport and energetics at atomic scale, otherwise presently beyond experimental reach.[30] In fact, MD simulations have been shown to
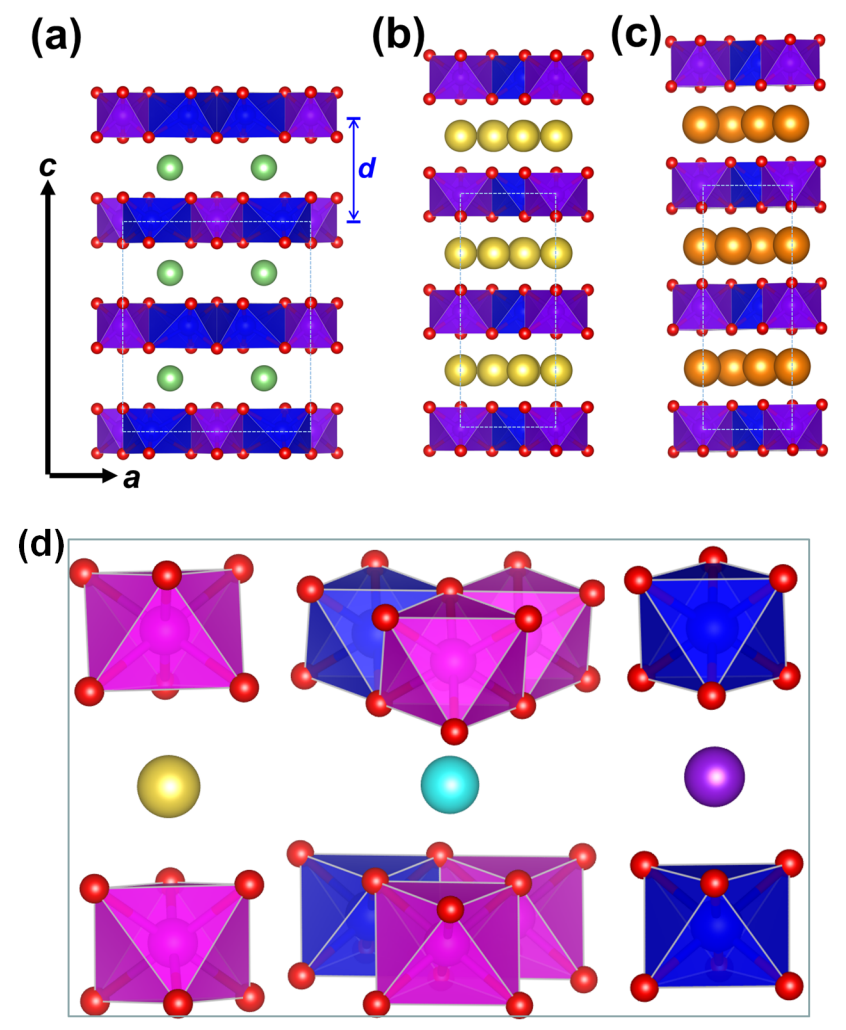

FIG. 1. A schematic of the $A_{2} \mathrm{Ni}_{2} \mathrm{TeO}_{6} \quad(A=\mathrm{Li}, \mathrm{Na}$ and $\mathrm{K})$ crystal structural framework whereby parallel slabs comprising $\mathrm{TeO}_{6}$ octahedra (in blue) and $\mathrm{NiO}_{6}$ octahedra (in magenta) are separated by alkali atoms. (a) Layered structure of $\mathrm{Li}_{2} \mathrm{Ni}_{2} \mathrm{TeO}_{6}$ (orthorhombic polymorph). $\mathrm{Li}$ atoms (in green) are located between slabs comprising $\mathrm{Te}$ (in blue) and $\mathrm{Ni}$ (in purple) in octahedral coordination with oxygen atoms (in red). (b) Layered structure of $\mathrm{Na}_{2} \mathrm{Ni}_{2} \mathrm{TeO}_{6}$ where $\mathrm{Na}$ atoms are depicted in yellow. (c) Layered structural framework of $\mathrm{K}_{2} \mathrm{Ni}_{2} \mathrm{TeO}_{6}$ (which is isostructural with $\mathrm{Na}_{2} \mathrm{Ni}_{2} \mathrm{TeO}_{6}$ ). $\mathrm{K}$ atoms are shown in brown. (d) Crystallographically distinct sites where alkali atoms reside in $\mathrm{Na}_{2} \mathrm{Ni}_{2} \mathrm{TeO}_{6}$ or $\mathrm{K}_{2} \mathrm{Ni}_{2} \mathrm{TeO}_{6}$, denoted as $A 1$ (in cyan), $A 2$ (in yellow) and $A 3$ (in purple) with partial alkali atom occupation. Their local polyhedral environments are also shown, for clarity. The inter-layer (inter-slab) distance is defined as ' $d$ '. Dashed lines denote the unit cell of $A_{2} \mathrm{Ni}_{2} \mathrm{TeO}_{6}(A=\mathrm{Li}, \mathrm{Na}$ and $\mathrm{K})$ and the axes for the $c$ - and $a$-axes parameters shown in (a) applies to all the structures shown.

accurately depict the structural and transport properties of previously reported energy materials such as our previous works on the physicochemical properties of $\mathrm{Na}$ based honeycomb layered tellurates (i.e., $\mathrm{Na}_{2} M_{2} \mathrm{TeO}_{6}$, where $M=\mathrm{Ni}, \mathrm{Zn}$, Co and Mg).[31-33] Moreover, these simulations have been instrumental in garnering insights into the diffusive properties of other materials[34] that have later on been experimentally confirmed (e.g., in $\left.\mathrm{Na}_{2} \mathrm{Ni}_{2} \mathrm{TeO}_{6}\right) \cdot[27,29]$ To accurately depict microscopic physicochemical properties of materials that are beyond experimental reach, it is therefore crucial to use reliable 
potential parameters that can effectively reproduce the structural and transport properties availed by experiment.

Thus, in this study we present an extensive MD study systematically performed using a refined set of interatomic potential parameters in $A_{2} \mathrm{Ni}_{2} \mathrm{TeO}_{6}$ (where $A$ $=\mathrm{Li}, \mathrm{Na}$, or $\mathrm{K})$. We focused on the fully discharged $A_{2} \mathrm{Ni}_{2} \mathrm{TeO}_{6}$ phases, since the crystallographic experimental data for the charged states of $A_{2} \mathrm{Ni}_{2} \mathrm{TeO}_{6}$ (i.e., $\left.A_{\mathrm{x}} \mathrm{Ni}_{2} \mathrm{TeO}_{6}(x<2)\right)$ are lacking in literature. Nonetheless, distinct from most layered oxides, it is known that $A_{2} \mathrm{Ni}_{2} \mathrm{TeO}_{6}$ presents a finite number of vacancies, even in the fully discharged state $(x=2)$. [ $7,10,15,18,21,28]$ The MD simulations demonstrate the effectiveness of the Vashishta-Rahman form of the interatomic potential,[35] reproducing various structural and transport properties of this class of materials. Further, we predict that an increase in the inter-layer distance results in improved microscopic transport of cations through diffusion. As such, the larger ionic radii of $\mathrm{K}$ and $\mathrm{Na}$ relative to $\mathrm{Li}$ engenders an exponential enhancement of the cationic conduction of $\mathrm{K}_{2} \mathrm{Ni}_{2} \mathrm{TeO}_{6}$ and $\mathrm{Na}_{2} \mathrm{Ni}_{2} \mathrm{TeO}_{6}$, in comparison with $\mathrm{Li}_{2} \mathrm{Ni}_{2} \mathrm{TeO}_{6}$. Through these investigations, we also connect the wider bottleneck radius formed by oxygens in cationic migration path for the larger mobile cations within the frameworks to the improved cationic diffusion experimentally observed in honeycomb layered oxides.

\section{METHODOLOGY}

\section{A. Interatomic Potential}

The previously reported Vashishta-Rahman form of the interatomic potential[35] is employed alongside parameters to produce the structural and dynamical properties of a series of honeycomb layered oxides, namely $\mathrm{Na}_{2} M_{2} \mathrm{TeO}_{6}(M=\mathrm{Ni}, \mathrm{Zn}, \mathrm{Co}$ and $\mathrm{Mg}),[31]$ as follows; [35]

$$
V_{i j}\left(r_{i j}\right)=\frac{q_{i} q_{j}}{r_{i j}}+\frac{A_{i j}\left(\tilde{\sigma}_{i}+\tilde{\sigma}_{j}\right)^{n_{i j}}}{r_{i j}^{n_{i j}}}-\frac{P_{i j}}{r_{i j}^{4}}-\frac{C_{i j}}{r_{i j}^{6}},
$$

where $q_{i}$ represents the charge and $\tilde{\sigma}_{i}, \tilde{\sigma}_{j}$ the ionic radius of the $i$-th, $j$-th ion. The parameters, $A_{i j}, P_{i j}$ and $C_{i j}$ describe the overlap-repulsion energy of the electron clouds, the (average) charge dipole interactions and the dispersion constant between ion pairs $i$ and $j$, respectively. Compared to the more popular Born-Mayer (Buckingham)[36] and Lennard-Jones potentials,[37] the Vashishta-Rahman potential has a softer overlap repulsion $\left(1 / r^{n}\right.$, where $n=11,9$ or 7 for cation-cation, cationanion and anion-anion pairs, respectively) in particular, between the anion pairs. Other related parameters, not available in literature, were determined using empirical fitting to attain the experimentally reported bond lengths $(\mathrm{Ni}-\mathrm{O}, \mathrm{Te}-\mathrm{O}$, and $\mathrm{O}-\mathrm{O})$ of $A_{2} \mathrm{Ni}_{2} \mathrm{TeO}_{6}$, where $A=\mathrm{Li}$, $\mathrm{Na}$, or K (Supplemental Material, Table SI). After sta- bilising the framework structure, the $A-A$ and $A-\mathrm{O}$ parameters were refined to reproduce the experimentally reported cationic hopping activation energy, where A and $\mathrm{O}$ play the roles of the cations and framework anions respectively.[31, 34, 38, 39]

\section{B. Computational Details}

Employing the optimised set of parameters listed in Table S1, a series of MD simulations were carried out in the temperature range of $500 \mathrm{~K}$ to $700 \mathrm{~K}$ with constant temperature maintained at $25 \mathrm{~K}$ intervals, and zero atmospheric pressure. The Parrinello-Rahman isobaricisothermal (NPT) MD method,[40] which allows for changes in the simulation box sizes whilst keeping angles fixed was used. The temperatures and pressure in the system were controlled using thermostatting and barostatting techniques whereby some dynamic variables are coupled with particle velocities and simulation box dimensions. Simulations commenced from the structures derived from the respective X-ray diffraction patterns wherein the $\mathrm{Li}_{2} \mathrm{Ni}_{2} \mathrm{TeO}_{6}$ simulation supercell was characterised by $3 \times 5 \times 2$-unit cells comprising 1320 atoms in a orthorhombic symmetry and the $A_{2} \mathrm{Ni}_{2} \mathrm{TeO}_{6}(A=$ $\mathrm{Na}$, and $\mathrm{K}$ )-systems consisted of 1100 atoms in $5 \times 5$ $\times 2$ rhombohedral unit cells. For $A=\mathrm{Na}$ and $\mathrm{K}$, several partially occupied crystallographic sites were identified. In the MD simulations, the respective $\mathrm{Na}$ and $\mathrm{K}$ ions were placed at the $A 2$ sites of the starting structure to avoid strong cation-cation repulsion. For clarity to readers, the crystallographically distinct sites $(A 1, A 2$ and $A 3)$ in the layered frameworks of $\mathrm{Na}_{2} \mathrm{Ni}_{2} \mathrm{TeO}_{6}$ and $\mathrm{K}_{2} \mathrm{Ni}_{2} \mathrm{TeO}_{6}$ are shown Figure 1 . A time step of 1 fs was applied to the Velocity Verlet algorithm to solve the Newton's equations of motion. A typical run-time of $6 \mathrm{~ns}$ or longer was used, with trajectory samples stored at intervals of $200 \mathrm{fs}$ for detailed analyses. In order to guarantee the thermodynamic convergence properties and ensure that the system size is adequate, a few longer run-time simulations of $100 \mathrm{~ns}$ as well as simulations on larger systems consisting of $6 \times 6 \times 3$-unit cells were additionally performed, respectively. Periodic boundary conditions in all three directions, and the Ewald summation technique for the convergence of long-range Coulombic interactions were applied using the LAMMPS software package.[41] A cut-off distance of $10 \AA$ was used for both the shortrange interactions and the short-range part of the Ewald summation. Canonical MD (NVT-MD) simulations were further performed using the average cell parameters obtained from NPT-MD, as NPT-MD modifies the kinetics to satisfy constant temperature and pressure conditions by sacrificing rigorous kinetic evolution. Nonetheless, we also performed microcanonical (NVE)-MD simulations for $\mathrm{K}_{2} \mathrm{Ni}_{2} \mathrm{TeO}_{6}$ and compared it with our NVT-MD simulation results.

Additionally, we performed two sets of MD simulation corresponding to the following two scenarios: (i) a se- 
ries of NVT-MD simulations performed at $600 \mathrm{~K}$ with progressively increased inter-slab distances (only $c$-axis parameters) along with constant $a, b$-axes parameters, and ionic radius in order to understand the influence of inter-layer distance decoupled from ionic radius. (ii) A series of NVT-MD simulations conducted at $600 \mathrm{~K}$ with progressively increased cationic sizes, namely $\tilde{\sigma}(1.20 \AA$ to $1.30 \AA$ at $0.05 \AA$ intervals), whilst keeping the interlayer distance fixed to correspond with the ionic radius of $\mathrm{K}$ (i.e., $1.38 \AA$ ), in order to understand the role of bottleneck radius formed by surrounded oxygen atoms. The aforementioned assumptions significantly divert from the already experimentally-verified correlation of inter-layer distance with ionic radius. Nonetheless, we should mention here that, for instance, subjecting this class of honeycomb layered materials to (positive) pressure corresponds to fixing the ionic radius whilst varying the inter-layer distance in accordance with scenario (i) above, whereas the converse (fixing the inter-layer distance and varying the ionic radius), in accordance with scenario (ii) above corresponds to expanding the material (i.e., inducing negative pressure). Consequently, conventional experimental results with unpressurised materials correspond to the zero-pressure scenario, where the inter-layer distance is strictly correlated with the ionic radius of the cations. As far as we can tell, experiments with finite pressure parameters have not yet been explored in experiments and simulations of this class of honeycomb layered oxides despite being standard in condensed matter experimental physics such as high-temperature superconducting materials where pressure modifies the transition temperature of the material and hence the conductivity, amongst other novelties.[42]

In the case of the $A_{2} \mathrm{Ni}_{2} \mathrm{TeO}_{6}$, pressure effects pertinent to the increase of inter-layer distance at fixed cationic size could be experimentally explored through chemical means, via the equation of state for the layered material. For instance, in a separate work, [43] two of the authors in this paper have conjectured that the curvature of the $a b$ plane in these materials correlates with the number of cations/vacancies and/or the topology of the surface, which in turn requires the curvature to be proportional to the energy density (or more succinctly the number per unit volume) of the diffusing cations. Typically, the number per unit volume, $\rho$ at thermodynamic equilibrium is related to pressure, $P$ by an equation of state.[44] This avails the avenue to chemically change $\rho$ or the curvature and hence $P$ in the material. A more rigorous discussion has been appended in the Supplemental Material, section IB. In the simulation, we additionally point out that we fixed the inter-layer distance and separately the ionic radius to that of the $\mathrm{K}$ system as it possesses the widest inter-layer distance and the largest ionic radius amongst the honeycomb layered nickel tellurates.

\section{Estimation of key quantities}

Following Einstein's relation, the self-diffusion coefficient of $\mathrm{Na}^{+}$is calculated from mean square displacement (MSD) for the two-dimensional transport as,

$$
\begin{array}{r}
\operatorname{MSD}=\frac{1}{N_{t}}\left\langle\sum_{j=1}^{N_{t}}\left(\vec{r}_{j}(t)-\vec{r}_{j}(0)\right)^{2}\right\rangle, \\
D=\lim _{t \rightarrow \infty} \frac{\mathrm{MSD}}{4 t},
\end{array}
$$

where $N_{t}$ denotes the total number of atoms in the system, $\vec{r}_{j}(\mathrm{t})$ is the position vector of the $j^{\text {th }}$ ion at time $t$ and the angular bracket indicates average over several time origins. Note that the MSD trends against time remain unchanged for both NVT and NVE simulations, as is shown in Figure S1. The diffusion coefficient, $D$, depends on temperature $(T)$ following Arrhenius equation,

$$
D=D_{0} \exp \left(\frac{-E_{\mathrm{a}}}{k_{\mathrm{B}} T}\right),
$$

where $D_{0}$ is the pre-exponential factor, $E_{\mathrm{a}}$ represents the activation energy of ion hopping, and $k_{\mathrm{B}}$ is the Boltzmann constant. The potential energy of individual cations is calculated as,

$$
V_{i}=\frac{1}{2} \sum_{j=1}^{N_{t}} V_{i j}
$$

where $V_{i j}$ is the interaction potential given in eq. (1), such that the total potential energy of the system is,

$$
V_{t}=\sum_{i=1}^{N_{t}} V_{i}
$$

Finally, the relative free energy (as seen by individual cations), $\Delta F$ can be measured relative to another system of cations at the location on the $a b$ plane exhibiting the maximum cation population density, $p_{\max }$ by the formula (Supplemental Material, section IA),

$$
-k_{\mathrm{B}} T \ln \left(\frac{p}{p_{\max }}\right)=\Delta F \simeq \Delta V-T \Delta S,
$$

where $p$ represents the population density function (occupancy) of the cations, $p_{\max }$ is the maximum value of the population density function, $\Delta V$ is the difference in potential energy of individual Also note that the population densities of cations remain unchanged for both NVE- and NVT-MD cases (Figure S2).

\section{RESULTS AND DISCUSSION}

\section{A. Framework Structure}

As shown in Figure 1, the $A_{2} \mathrm{Ni}_{2} \mathrm{TeO}_{6}$ compounds, where $A=\mathrm{Li}, \mathrm{Na}$, and $\mathrm{K}$, have a layered structure of $A$ cations sandwiched between transition metal 
TABLE I. A comparison of average cell parameters (in $\AA$ ) calculated from isobaric-isothermal (NPT) MD simulations performed at $300 \mathrm{~K}$ for the $A_{2} \mathrm{Ni}_{2} \mathrm{TeO}_{6}(A=\mathrm{Li}, \mathrm{Na}$, and $\mathrm{K})$ with reported experimental results (abbreviated as 'Expt.').

\begin{tabular}{|c|ccc|ccc|ccc|}
\hline $\begin{array}{c}\text { Cell } \\
\text { Parameters }\end{array}$ & \multicolumn{4}{|c|}{$\mathrm{Li}$} & \multicolumn{4}{c|}{$\mathrm{Na}$} & \multicolumn{4}{c|}{$\mathrm{K}$} \\
\hline \hline$a$ & 8.992 & 8.700 & 3.0 & 5.207 & 5.211 & 0.1 & 5.261 & 5.211 & 0.9 \\
$b$ & 5.147 & 4.975 & 3.0 & 5.207 & 5.211 & 0.1 & 5.261 & 5.211 & 0.9 \\
$c$ & 10.169 & 9.829 & 3.0 & 11.1558 & 11.167 & 0.1 & 12.467 & 12.349 & 0.9 \\
\hline \hline
\end{tabular}

TABLE II. Average interatomic distances in angstrom $(\AA)$ for the $\mathrm{Te}-\mathrm{O}$ and $\mathrm{Ni}-\mathrm{O}$ octahedra based on NPT-MD simulations conducted at $300 \mathrm{~K}$.

\begin{tabular}{|l|rr|rr|rr|}
\hline & \multicolumn{2}{|c|}{ Li } & \multicolumn{2}{|c|}{ Na } & \multicolumn{2}{|}{ K } \\
& Expt.[12] & MD & Expt.[21] & MD & Expt.[15] & MD \\
\hline Te-O & 1.9931 .990 & 1.971 & 1.990 & 1.951 & 1.990 \\
$\mathrm{Ni}-\mathrm{O}$ & 2.035 & 2.020 & 2.148 & 2.070 & 2.112 & 2.070 \\
\hline
\end{tabular}

slabs of $\mathrm{TeO}_{6}$ and $\mathrm{NiO}_{6}$ octahedra arrayed in a honeycomb fashion. Experimental reports have shown $\mathrm{Na}_{2} \mathrm{Ni}_{2} \mathrm{TeO}_{6}$ and $\mathrm{K}_{2} \mathrm{Ni}_{2} \mathrm{TeO}_{6}$ to embody hexagonal crystal symmetries, $[15,21]$ whilst $\mathrm{Li}_{2} \mathrm{Ni}_{2} \mathrm{TeO}_{6}$ is known to entail an orthorhombic and monoclinic crystal symmetries contingent on the synthetic protocols.[12] Moreover, all the octahedral layers in the $\mathrm{Na}_{2} \mathrm{Ni}_{2} \mathrm{TeO}_{6}$ and $\mathrm{K}_{2} \mathrm{Ni}_{2} \mathrm{TeO}_{6}$ are seen to be identical, whereas a slight shift between the top and bottom layer around the conduction plane is observed in the case of $\mathrm{Li}_{2} \mathrm{Ni}_{2} \mathrm{TeO}_{6}$ (as shown in Figures 1(a), 1(b) and 1(c)). The layers are held together along the $c$-axis by Van der Waals forces and by interactions mediated via $A$ atoms occupying their inter-layers. The refined set of interatomic potential parameters retains these structural features in conformity with experimental findings. The average cell parameters of $A_{2} \mathrm{Ni}_{2} \mathrm{TeO}_{6}$ calculated from NPT-MD simulations at $300 \mathrm{~K}$ are placed alongside the room-temperature experimental values in Table I, where the calculated $c$ axis parameter (given the crystallographic arrangement of the layers in the $c$-axis direction) increases upon substitution of the $A$ atom species in the order of $\mathrm{Li}, \mathrm{Na}$ and $\mathrm{K}$, respectively, in corroboration with experimental observations.[12, 15, 21] The progressive increase noted in the cell parameters of this family of materials is ascribed to increases in cation sizes in the order of $\mathrm{Li}, \mathrm{Na}$ and $\mathrm{K}$ as a result of intensified ionic repulsion inside the layers as prescribed in the presence of larger sized cations. It should be noted that the deviations from experimental values in all cases are well within $3 \%$.

For a comparison of the local structures, radial distribution functions, $g(r)$, between the framework ion pairs, $\mathrm{Ni}-\mathrm{O}, \mathrm{Te}-\mathrm{O}$, and $\mathrm{O}-\mathrm{O}$, obtained from NPT-MD simulations at $300 \mathrm{~K}$ for the $A_{2} \mathrm{Ni}_{2} \mathrm{TeO}_{6}$ compounds $(A=\mathrm{Li}$, $\mathrm{Na}$, and $\mathrm{K}$ ) are furnished in Figure 2 . For clarity, the radial distribution functions calculated from the respec- tive X-ray diffraction (XRD) structures[12, 15, 21, 28] are also produced in the form of bar-plots. Noteworthy is that $\mathrm{Li}_{2} \mathrm{Ni}_{2} \mathrm{TeO}_{6}$ shows structural disorders, resulting in the emergence of several XRD peaks compared to $\mathrm{Na}_{2} \mathrm{Ni}_{2} \mathrm{TeO}_{6}$ and $\mathrm{K}_{2} \mathrm{Ni}_{2} \mathrm{TeO}_{6}$. Except for the thermal broadening, all the calculated peaks from MD simulations are reasonably consistent with their corresponding $g(r)$, calculated for the XRD structure.For Te and $\mathrm{Ni}$, exactly six oxygen coordination numbers are found within the 1st neighbouring distance, forming $\mathrm{NiO}_{6}$ and $\mathrm{TeO}_{6}$ octahedra. The average $\mathrm{Te}-\mathrm{O}$ and $\mathrm{Ni}-\mathrm{O}$ distances of the octahedra for $A=\mathrm{Li}, \mathrm{Na}$, and $\mathrm{K}$, calculated from $\mathrm{MD}$ simulations (listed in Table II), also show good agreement with the reported XRD structure. Similarly, the radial distribution functions obtained from the other simulations performed under different conditions (see the METHODOLOGY section II) revealed that in all cases, the respective crystal structures were retained, affirming that the refined set of interatomic parameters successfully reproduce the structure.

\section{B. Ionic Conductivity}

The $A$ cations are located in the $a b$-plane between the polyhedral layers and with loose coordinations to the framework layers which constitute a large number of cationic sublattices, specifically for $A=\mathrm{Na}$, and K. These sublattices facilitate high ionic diffusion, in accordance with eq. (2a) and ascertained by the mean square displacements (MSD) of the cations plotted against time (Figure 3(a)). On the other hand, the closely packed octahedral layers parallel to the $a b$-plane restrict the cationic motion along the $c$-axis direction of the cell, as reflected in the inset of Figure 3(a) (less than $0.2 \AA[2]$ ). Therefore, it can be deduced that cationic mobility is restricted within the sublattices oriented parallel to the $a b$-plane, rendering the diffusion to be highly anisotropic (viz., two-dimensional). The MSD of the $A$ ions is noted to increase drastically with increase in cationic size in the order of $\mathrm{Li}, \mathrm{Na}$ and $\mathrm{K}$. Figure 3(b) displays the diffusion coefficient, which was calculated from the slope of Figure 3(a) using eq. (2a), as a function of inter-layer distance. It is worth noting that the diffusion coefficient unequivocally follows the exponential dependency of the inter-layer distance, whereas the experimental activation energy diminishes linearly with increasing inter-layer dis- 
(a)

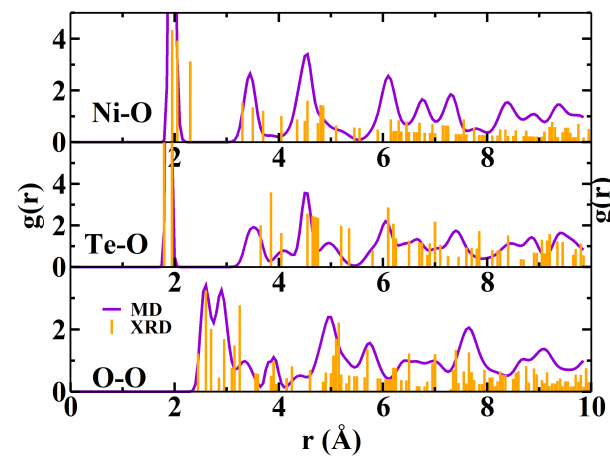

(b)

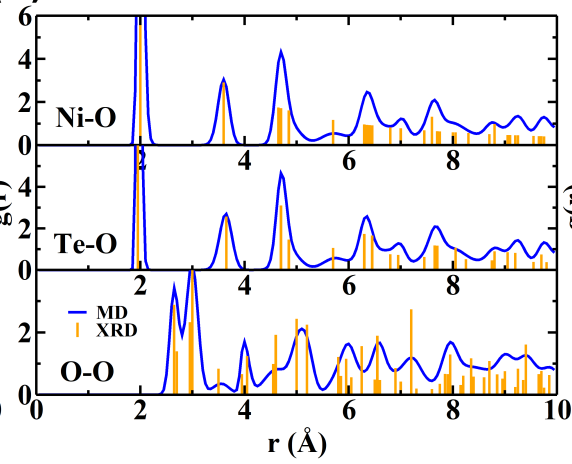

(c)

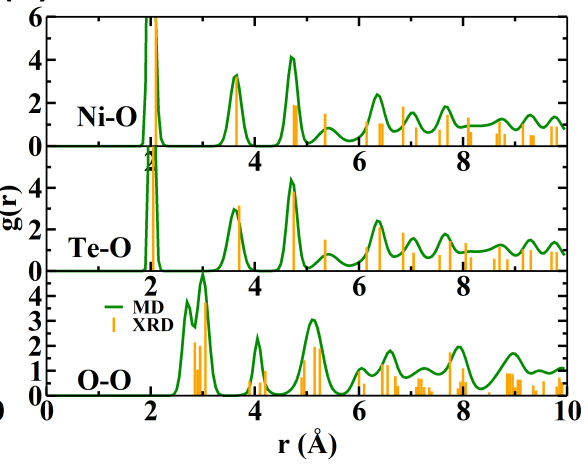

FIG. 2. Radial distribution function $(g(r))$ between selected ion pairs (namely, Ni-O, Te-O and O-O) at $300 \mathrm{~K}$ for (a) $\mathrm{Li}_{2} \mathrm{Ni}_{2} \mathrm{TeO}_{6}$, (b) $\mathrm{Na}_{2} \mathrm{Ni}_{2} \mathrm{TeO}_{6}$ and (c) $\mathrm{K}_{2} \mathrm{Ni}_{2} \mathrm{TeO}_{6}$. For clarity, the vertical bars shown in orange are the corresponding radial distribution functions derived from X-ray diffraction analyses.[12, 15, 21, 28] The bar heights are reduced for easy visualisation.

(a)

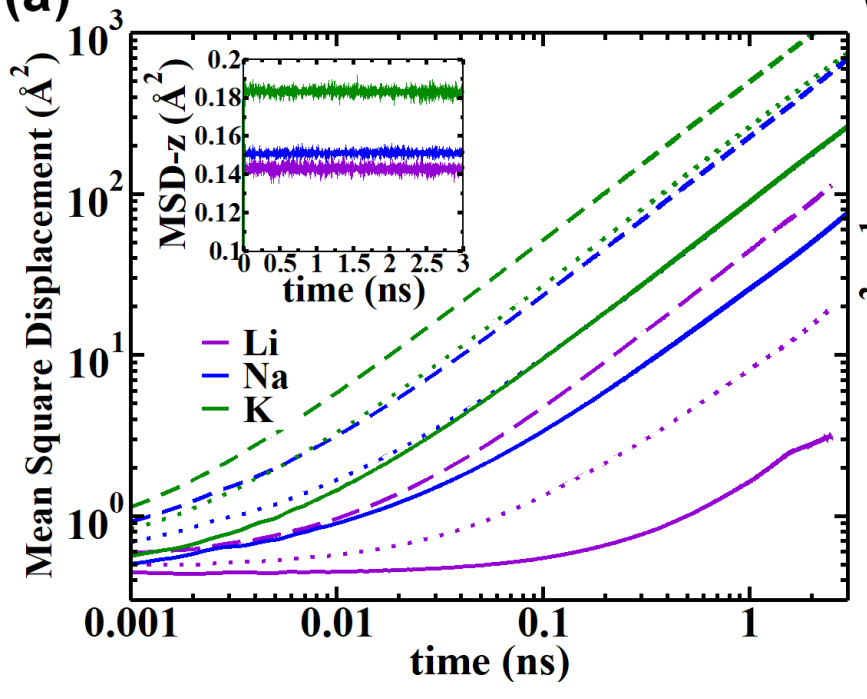

(b)

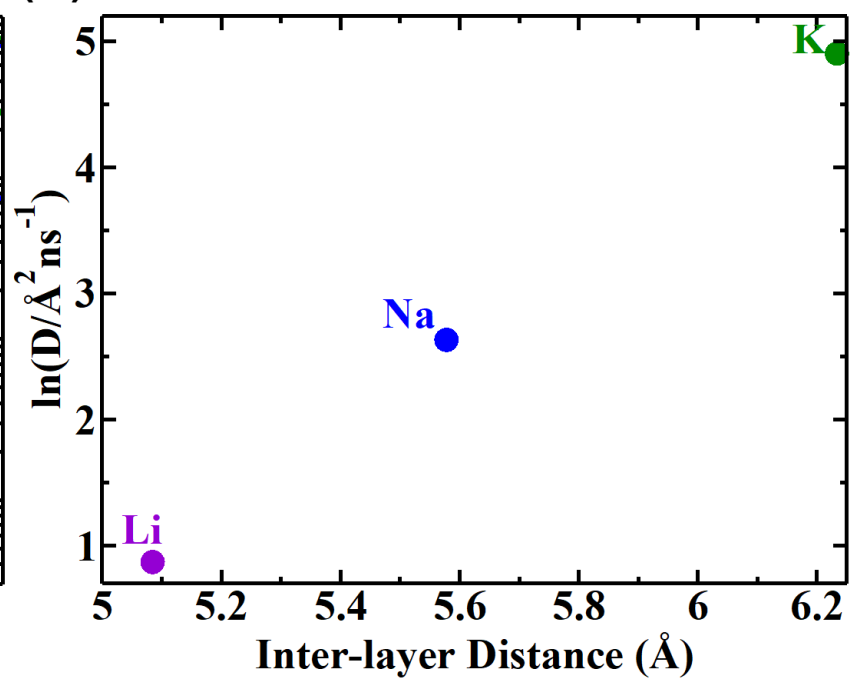

FIG. 3. (a) Logarithmic plots of mean square displacement (MSD) of cations against time in $A_{2} \mathrm{Ni}_{2} \mathrm{TeO}_{6}(A=\mathrm{Li}, \mathrm{Na}$ and $\mathrm{K}$ ) based on canonical (NVT) MD simulations. The continuous, dotted and dashed lines are for 500, 600 and $700 \mathrm{~K}$, respectively. Inset shows the MSD of cations (along the $c$-axis direction (MSD- $z$ )) at $600 \mathrm{~K}$. These results demonstrate that cationic mobility is restricted along the $a b$-plane, rendering the diffusion to be highly anisotropic. (b) A logarithmic plot of the diffusion coefficient $(D)$ as a function of the inter-layer distance (calculated from the gradient of the MSD graphs in (a) for $600 \mathrm{~K}$ ).

tances as is displayed in Figure 3(b).[15, 21]

The logarithmic plots of $D$ versus inverse temperature $(1000 / T)$ are displayed in Figure 4(a). According to eq. (3), $\ln D=\ln D_{0}-E_{\mathrm{a}} / k_{\mathrm{B}} T$ corresponds to the equation of a straight line, where $-E_{\mathrm{a}} / k_{\mathrm{B}}$ is the slope and $\ln D_{0}$ the $y$-intercept. This implies that both the activation energy and the pre-exponential factor can be readily extracted from Figure 4(a). The activation energies were calculated using the diffusion constant (related to the diffusion coefficient in eq. (3)) instead of conductivity (Green-Kubo relation), an experimentally measurable quantity. It is worthy to note that conductivity, $\sigma$ at thermal equilibrium and diffusion constant, $D$ can be succinctly linked by $\sigma=q \mu \rho$ and $\mu=D / k_{\mathrm{B}} T$, where $\mu$ is mobility, $\rho$ is charge density and $q$ is the unit charge. This implies that both approaches are equivalent in particular regimes (Supplemental Material, section IC). However, even though the Green-Kubo formula appears the more straightforward way to make the connection with measurable quantities such as conductivity, simulations with it are known to yield infinitely slow decay (or what is referred to as 'long-time tail') in conserved hydrodynamic variables. [45] This includes large fluctuations in the calculated electrical conductivity at long times.[46-48] The 


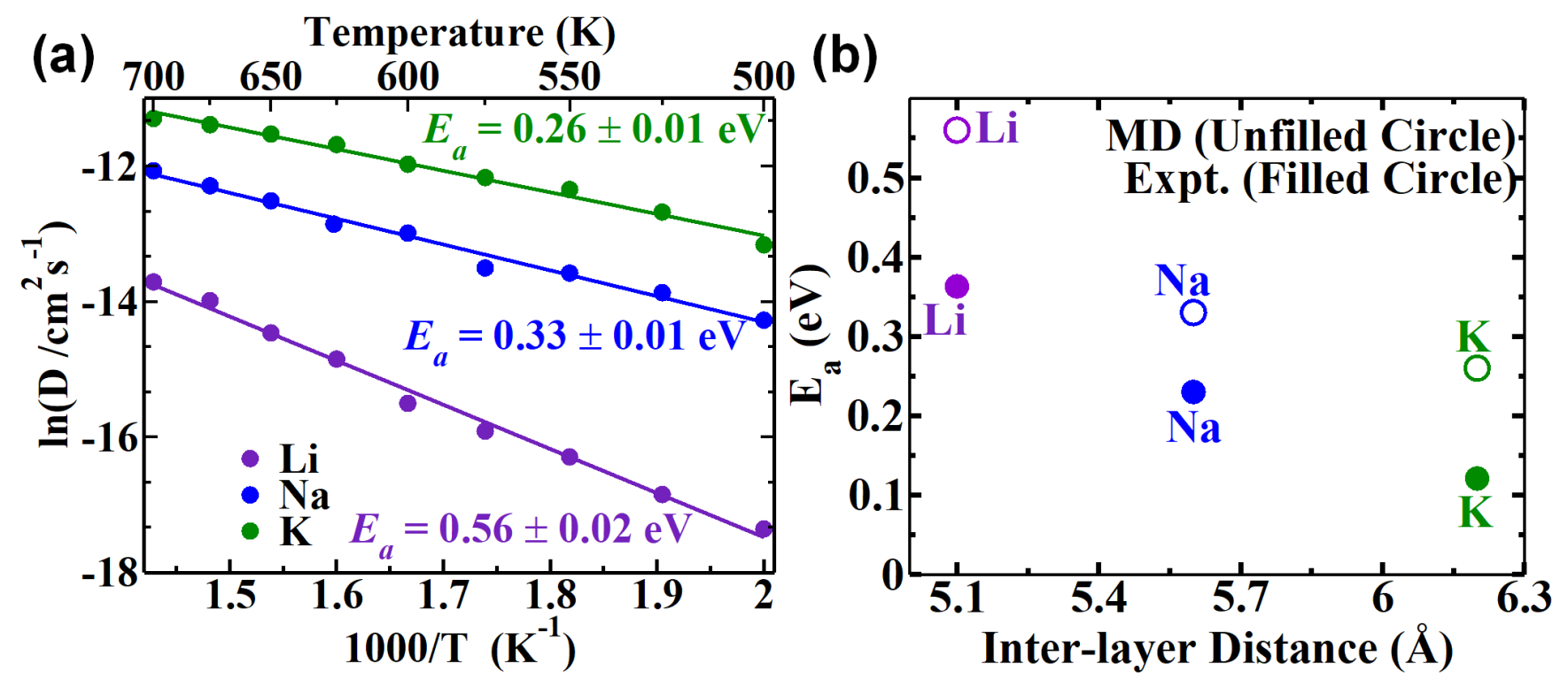

FIG. 4. (a) Logarithmic plots of the diffusion coefficient $(D)$ for alkali ions in $A_{2} \mathrm{Ni}_{2} \mathrm{TeO}_{6}(A=\mathrm{Li}$, Na and K) versus inverse temperature as extracted from molecular dynamics (MD) simulation results for the temperature range of 500-700 $\mathrm{K}$ at intervals of $25 \mathrm{~K}$. Errors in the attained activation energy values are determined from the standard deviation. The slopes for $\mathrm{Li}_{2} \mathrm{Ni}_{2} \mathrm{TeO}_{6}$, $\mathrm{Na}_{2} \mathrm{Ni}_{2} \mathrm{TeO}_{6}$ and $\mathrm{K}_{2} \mathrm{Ni}_{2} \mathrm{TeO}_{6}$ are respectively shown in purple, blue and green. (b) The activation energy values based on both experiment (abbreviated as Expt.) [21, 28, 43] and computation (MD simulations) against the inter-layer distances in $A_{2} \mathrm{Ni}_{2} \mathrm{TeO}_{6}(A=\mathrm{Li}, \mathrm{Na}$ and $\mathrm{K})$. Note that the correlation plots of experimental against the computed ionic conductivity and activation energy values have been listed, demonstrating univocally that the Vashishta-Rahman potential adopted for our MD computation captures, with high fidelity, the appropriate physics of ion diffusion in this class of honeycomb layered oxides

activation energy of $\mathrm{Li}_{2} \mathrm{Ni}_{2} \mathrm{TeO}_{6}$ is found to be highest $(0.56 \mathrm{eV})$ and lowest $(0.26 \mathrm{eV})$ for $\mathrm{K}_{2} \mathrm{Ni}_{2} \mathrm{TeO}_{6}$ amongst the three systems. A comparison of the activation energies calculated from the present MD simulations and reported experimental results is shown in Figure 4(b). Note that the MD results overestimate the activation energies for the three compounds compared to the reported ones. Usually, real material systems consist of several vacancies due to defects that lead to higher diffusion, which have not been considered here. Moreover, we have used the initial potential model of $\mathrm{Na}_{2} \mathrm{Ni}_{2} \mathrm{TeO}_{6}$ (with overestimated values); thus, the overestimation is propagated to all other materials. Nonetheless, the present MD simulation model successfully reproduces the diffusion trend observed in Li-, Na- and K-based honeycomb layered nickel-based tellurate systems. Therefore, this validates the utility of the Vashishta-Rahman form of the interatomic potential in reproducing the experimental physicochemical properties. The activation energy values reported from both experiments and MD simulations display an almost linear decaying behaviour with increasing inter-layer distances (as further shown in Figure 4(b)).

Alkali-ion diffusion in materials, in particular, entail the concomitant interplay between the inter-layer (interslab) distance and cationic size. In principle, a wider inter-slab distance reduces the interaction between the mobile cation and the ions in the transition metal slab, culminating in higher cationic diffusion (as identified in Figure 3(b), by the increase of cationic diffusion (diffusivity) coefficient $(D)$ with increase in inter-layer distance. Consequently, due to the correlation of inter-layer distance with ionic radius in unpressurised materials, the cationic diffusion (diffusivity) is expected to increase with increasing cationic size. Therefore, it is difficult to ascertain the role of cationic size since the enhancement of diffusivity in $\mathrm{K}$ systems can also be attributed to the increased inter-layer distance. Fortunately, it is possible in theoretical simulations to decouple the inter-layer distance from the cation size and treat them independently of each other even though they are typically correlated in unpressurised systems. Therefore, in the MD simulations, the $c$-axis parameter, which corresponds to the inter-layer distance, is increased whilst the cation size is kept constant. The $c$-axis parameter was progressively increased from 11.3 to $13.3 \AA$ at intervals of $0.2 \AA$, to cover the inter-layer distance range from $\mathrm{Li}$ to $\mathrm{K}$. The ionic radius was kept constant at the value corresponding to that of $\mathrm{K}$ (i.e., $1.38 \AA$ ), as $\mathrm{K}$-ions have the largest ionic radius reported so far for this family of honeycomb layered oxides. As can be seen in the plot of the inter-slab distribution (furnished as Figure S3 in the Supplementary Information), increasing the $c$-axis parameter basically increases the inter-slab distance, $d$ (i.e., $d=c / 2$ ) 


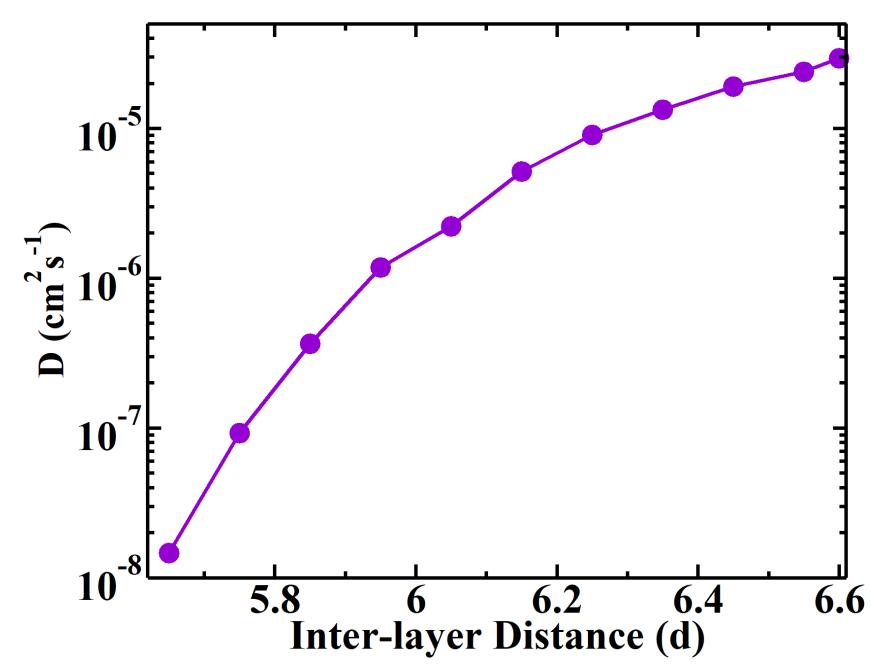

FIG. 5. Diffusion coefficient $(D)$ of cations in $\mathrm{K}_{2} \mathrm{Ni}_{2} \mathrm{TeO}_{6}$ from NVT-MD simulation at $600 \mathrm{~K}$ acquired by systematically increasing the inter-slab distances (viz., by only increasing the $c$-axis parameters) whilst fixing the $a$ - and $b$-axes parameters.

without forming other structural artifacts in the MD simulation, such as undulation. However, it is important to note that different size cations engender different interlayer distances to maintain structural stability. Thus, through MD simulations, it is possible to study microscopic phenomena beyond the reach of practical experiments.

Accordingly, the inter-layer distances below the ionic radius of lithium were not considered because lithium cations are the smallest cations that embody the present honeycomb layered framework. Moreover, the cationic diffusion was observed to diminish with decrease in the inter-layer distance ( $c$-axis parameter), as shown in Figure $\mathrm{S} 4$; indicative that inter-layer distances smaller than $11.3 \AA$, would result in unfavourable cationic diffusion. Notably, the diffusivity trend significantly deviates from exponential dependency of inter-layer distances as shown in Figure 5, whereby the logarithmic of diffusion coefficient plot shows a converging nature at higher inter-layer distances. Although this might be a consequence of the current simulation conditions, where the $a$ - and $b$-axes parameters and the ionic sizes are kept constant, it might also be an indicator that larger inter-layer distances do not necessarily result in higher cationic diffusion presumably as a result of structural instability or diminished inter-layer interactions.

\section{Microscopic mechanisms of cationic transport}

Although the aforementioned results are already illuminating, it is imperative to take into consideration the atomistic mechanisms governing the cations confined within the sublattices along the $a b$-plane in order to accurately discern the nature of the enhanced diffusion in $\mathrm{Na}_{2} \mathrm{Ni}_{2} \mathrm{TeO}_{6}$ and $\mathrm{K}_{2} \mathrm{Ni}_{2} \mathrm{TeO}_{6}$ compared to $\mathrm{Li}_{2} \mathrm{Ni}_{2} \mathrm{TeO}_{6}$. On that account, three different cationic sites were identified from experimental studies inside the conduction plane of $A_{2} \mathrm{Ni}_{2} \mathrm{TeO}_{6}(A=\mathrm{Na}$ and $\mathrm{K})$ in each inter-layer of a unit cell, labelled as $A 1, A 2$ and $A 3$, with a multiplicity of 3,2 , and 1 respectively.[15, 21, 27, 28] (For clarity to readers, $A 1, A 2$ and $A 3$ are crystallographically distinct sites.) These sites are the centre of trigonal prisms formed by oxygen atoms, as shown in Figure 1. The octahedral layers include tetrahedral voids (or 'holes') formed between two neighbouring $\mathrm{NiO}_{6}$ and $\mathrm{TeO}_{6}$ octahedra with the $A 1$ site located mid-way between the two tetrahedral voids from the top and bottom layers. The $A 2$ sites are sandwiched between the triangular faces of $\mathrm{NiO}_{6}$ octahedra from the top and bottom layers, whereas the $A 3$ site is located between two $\mathrm{TeO}_{6}$ octahedra. However, the experimentally reported $\mathrm{Li}_{2} \mathrm{Ni}_{2} \mathrm{TeO}_{6}$ shows a different structure whereby the $\mathrm{NiO}_{6}$ octahedra are slightly deformed, and the layered stacking sequence is different from the $\mathrm{Na}_{2} \mathrm{Ni}_{2} \mathrm{TeO}_{6}$ and $\mathrm{K}_{2} \mathrm{Ni}_{2} \mathrm{TeO}_{6}$ which have the $\mathrm{TeO}_{6}$ octahedra at the top and bottom of the $A 3$-sites. The smaller inter-layer separation $(5.08 \AA)$ in $\mathrm{Li}_{2} \mathrm{Ni}_{2} \mathrm{TeO}_{6}$ (compared to $\mathrm{Na}_{2} \mathrm{Ni}_{2} \mathrm{TeO}_{6} \quad(5.57 \AA)$ and $\mathrm{K}_{2} \mathrm{Ni}_{2} \mathrm{TeO}_{6}(6.23 \AA)$ ), engenders a high Te-Te Coulombic repulsion along the $c$-axis direction which prevents the $\mathrm{TeO}_{6}$ octahedra from achieving a stable vertical orientation. Thus, unlike the $\mathrm{Na}$ and $\mathrm{K}$ systems, the $\mathrm{Li}$ cations orient themselves at the top and bottom of the octahedral voids in order to avoid the resultant strong repulsion at the $A 3$ site.

In order to understand cationic site preferences and hopping energy barriers between sites, the population density (number per unit area) pattern and average potential energy of the mobile cations were calculated in accordance with eq. (5) (detailed in the METHODOLOGY section II). Both the population density and potential energy in the conduction plane are projected onto a $2 \mathrm{D}$ grid on the $a b$-plane (with all the coordinates of the cations folded into a single cell). Figure 6 displays the population density, whilst Figure 7 shows the corresponding potential energy surface of cations in the order of $\mathrm{Li}$, $\mathrm{Na}$ and K (replicated over $2 \times 2$-unit cells for easy visualisation of their continuity). The Li population densities are mostly localised due to the deep potential wells with minima of $-3.2 \mathrm{eV}$ (Figures 6(a) and 7(a)), whereas the Na-substituted system exhibits well-connected cationic diffusion channels between the $A 1$ and $A 2$ sites (Figures $6(\mathrm{~b})$ and $7(\mathrm{~b}))$. Due to the aforementioned strong repulsion, there are no Na cations occupying the $A 3$ sites, which are located directly between the top and bottom $\mathrm{Te}-\mathrm{Te}$ octahedra along the $c$-axis direction. In the case of $\mathrm{Na}_{2} \mathrm{Ni}_{2} \mathrm{TeO}_{6}$, the potential wells are relatively shallow $(-2.6 \mathrm{eV})$ compared to the $\mathrm{Li}$ system. Conversely, $\mathrm{K}_{2} \mathrm{Ni}_{2} \mathrm{TeO}_{6}$ exhibits slightly different profiles (Figures $6(\mathrm{c})$ and $7(\mathrm{c}))$, characterised by the shallowest potential wells $(-2.2 \mathrm{eV})$ amongst the three systems. Thus, 
(a)

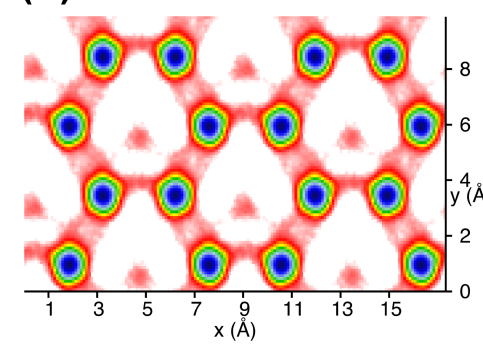

(b)

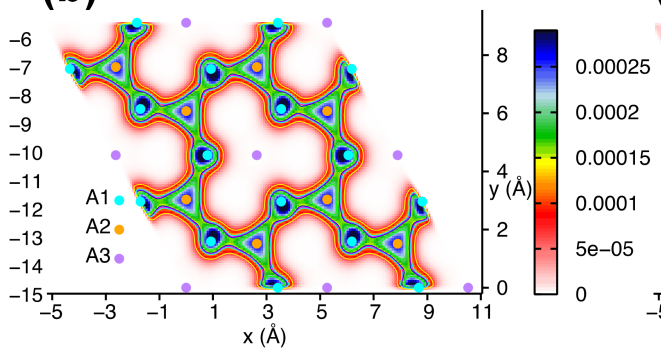

(c)

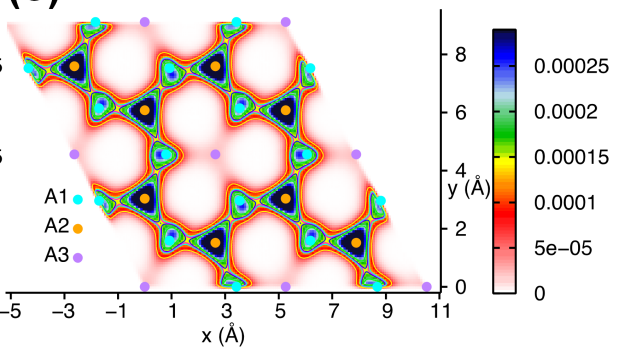

FIG. 6. Average individual population profiles of mobile $A$ cations for $A_{2} \mathrm{Ni}_{2} \mathrm{TeO}_{6}(A=\mathrm{Li}$, Na and K) derived from NVT-MD simulations at $600 \mathrm{~K}$ (mapped on to $2 \times 2$-unit cells). The population profiles for (a) $\mathrm{Li}_{2} \mathrm{Ni}_{2} \mathrm{TeO}_{6}$, (b) $\mathrm{Na}_{2} \mathrm{Ni}_{2} \mathrm{TeO}_{6}$ and (c) $\mathrm{K}_{2} \mathrm{Ni}_{2} \mathrm{TeO}_{6}$. Note that the colour bar in (a) in represented in a logarithmic scale for better visualisation. A common colour bar is used in both (b) and (c). The location of the crystallographically distinct alkali atom sites for $\mathrm{Na}_{2} \mathrm{Ni}_{2} \mathrm{TeO}_{6}$ and $\mathrm{K}_{2} \mathrm{Ni}_{2} \mathrm{TeO}_{6}$, namely $A 1, A 2$, and $A 3$, are shown in legends in (b) and (c). The population contours reflect that the preferred migration pathway of the cations is solely within the $A 1$ and $A 2$ sites (viz., $A 1-A 2-A 1-A 2 \cdots$ ). Note that the $\mathrm{Li}$ sites in $\mathrm{Li}_{2} \mathrm{Ni}_{2} \mathrm{TeO}_{6}$ are not marked, as their population density at $600 \mathrm{~K}$ deviates from that at room temperature in their crystallographic sites.

(a)

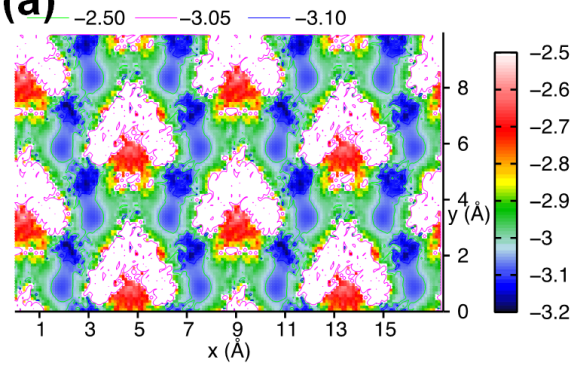

(b)

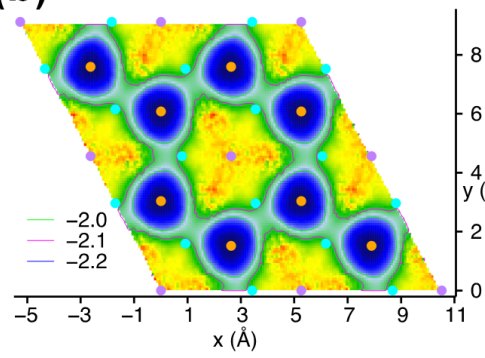

(c)

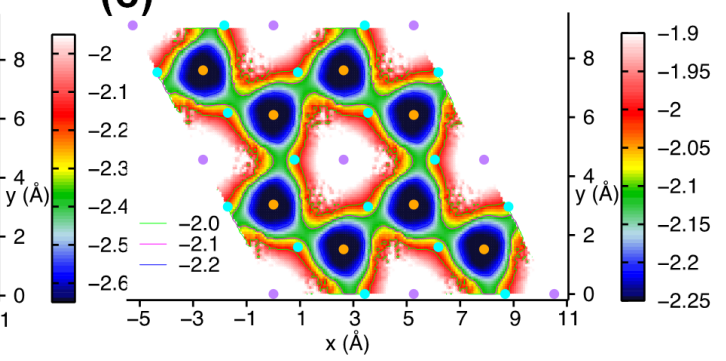

FIG. 7. Potential energy profiles of mobile $A$ cations for $A_{2} \mathrm{Ni}_{2} \mathrm{TeO}_{6}(A=\mathrm{Li}, \mathrm{Na}$ and $\mathrm{K})$ derived from NVT-MD simulations at $600 \mathrm{~K}$ (mapped on to $2 \times 2$-unit cells) using eq. (4). The potential energy profiles for (a) $\mathrm{Li}_{2} \mathrm{Ni}_{2} \mathrm{TeO}_{6},(\mathrm{~b}) \mathrm{Na}_{2} \mathrm{Ni}_{2} \mathrm{TeO}_{6}$ and (c) $\mathrm{K}_{2} \mathrm{Ni}_{2} \mathrm{TeO}_{6}$. The location of the crystallographically distinct alkali atom sites for $\mathrm{Na}_{2} \mathrm{Ni}_{2} \mathrm{TeO}_{6}$ and $\mathrm{K}_{2} \mathrm{Ni}_{2} \mathrm{TeO}_{6}, \mathrm{namely}$ $A 1$ (cyan), $A 2$ (orange), and $A 3$ (purple), are shown in legends in (b) and (c). The average individual population profiles in Figure 6 are related to the potential energy profiles via eq. (6). Thus, the difference in the two profiles can be attributed to a finite relative entropy, $\Delta S$ in the system.

even though the population density profile of cations in $\mathrm{K}_{2} \mathrm{Ni}_{2} \mathrm{TeO}_{6}$ is slightly different than of $\mathrm{Na}_{2} \mathrm{Ni}_{2} \mathrm{TeO}_{6}$, the path connectivity of $\mathrm{K}^{+}$is essentially the same as that simulated for $\mathrm{Na}^{+}$, indicating the same cation hopping route. The shallower potential profile in the $\mathrm{K}$ system attests to its considerably lower potential energy barrier, allowing for more facile shifts of $\mathrm{K}$ cations to and from the $A 1$ and $A 2$ sites compared to the Na system. The calculated potential energy difference from $A 1$ to $A 2$ is $0.25 \mathrm{eV}$; albeit a slightly different value than reported based on bond valency energy landscape (BVEL) summation.[18] Note that BVEL heavily relies on experimental data for tuning, thus compromising its accuracy and predictive power. Thus, the results in Figure 7 evince that the minima values of the potential energy increase (i.e., the potential becomes shallower) in the order of $\mathrm{Li}$, $\mathrm{Na}$ and $\mathrm{K}$, which also corresponds to the increasing order of the inter-layer distances, indicative that the potential energy barrier inhibiting the hopping of the cations be- tween sites reduces with inter-layer distance, leading to a drastically higher cationic diffusion. This was additionally affirmed by calculations of the profiles in relation to the progressively increasing inter-layer distances, as shown in Figure 5.

For a more detailed quantitative estimation of the role of the cationic occupancy and its associated (relative) free energy landscape, we considered the $2 \mathrm{D}$ population density distribution for progressively increasing $c$-axes parameters (Figure S5), and calculated the (relative) free energy (Figure S6(b)) from the population distribution (Figure S6(a)) along a straight-line path conjured up by an imaginary cylindrical axis with a radius of $0.8 \AA$ connecting the neighbouring $A 2$ and $A 1$ sites in accordance with eq. (6) (see the METHODOLOGY section II). Note that, the $\mathrm{Na}$ and $\mathrm{K}$ systems exhibit slightly differing $A 1$ and $A 2$ site occupancy, as is revealed by the population density profile shown in Figures 6(b) and 6(c) respectively. For instance, in the Na system, the 
population densities at the $A 1$ and $A 2$-sites are almost identical whereas in the $\mathrm{K}$ system, the population at the $A 2$-sites is significantly higher than the $A 1$-sites. Remarkably, (relative) free energy distribution calculations from their respective population density distributions revealed that although $\mathrm{K}$ system exhibits a lower activation energy than the $\mathrm{Na}$ system, the (relative) free energy barrier for $\mathrm{K}$ ion diffusion $(0.07 \mathrm{eV})$ is higher than its $\mathrm{Na}$ counterpart $(0.04 \mathrm{eV})$ (Figure S6(b)). This juxtaposed behaviour can be ascribed to the site topology and the connectivity of the two systems. Moreover, as illustrated in Figure 6(b, c) and Figure 7(b, c), the $A 1$ sites are connected by two $A 2$ sites, whereas $A 2$ sites are connected by three $A 1$ sites implying that a cation occupying an $A 1$ site has two possible paths (corresponding to a configuration entropy of $\sim k_{\mathrm{B}} \ln 2$ ), whereas it finds three possible paths if it occupies an $A 2$-site (configuration entropy of $\left.\sim k_{\mathrm{B}} \ln 3\right)$. Therefore, because the $\mathrm{Na}$ system is characterised by higher population densities at the $A 1$-sites, cationic diffusion from $A 2$ to $A 1$ will be restricted by the lower availability of paths (lower configuration entropy). Conversely, a lower population density implies a higher number of cationic vacancies. Hence the vacancies at $A 2$ are larger than at $A 1$, and by extension, Na cations can hop from $A 1$ to $A 2$, corresponding to a 'hole' hopping from $A 2$ to $A 1$ sites, where the 'holes' also have three available $A 2$ to $A 1$ pathways (configuration entropy of $\sim k_{\mathrm{B}} \ln 3$ ), and hence contributes an opposite diffusion current. Since the pathway with the larger configuration entropy contributes to the bulk of the diffusion current in the system, it implies that in the $\mathrm{Na}$ system, the 'holes' will contribute the bulk of the diffusion current, thus offsetting any low configuration entropy restrictions to the diffusion current imposed on the cations in the $A 1$ sites. Likewise, the lower population density of $\mathrm{K}$ cations at $A 1$ sites compared to $A 2$ sites and the lower configuration entropy restricts the 'holes' from hopping from the $A 1$ sites to the $A 2$ sites whilst the higher population density and configuration entropy of the $\mathrm{K}$ cations at the $A 2$ compared to the $A 1$ site avails more avenues for the $\mathrm{K}$ cations to hop from $A 2$ sites to $A 1$ sites, implying that the bulk of the current in $\mathrm{K}$ systems is as a result of the high configuration entropy of the cations at $A 2$ site. Thus, this cation-'hole' picture also implies that the cation occupancy/vacancies and population density distributions alone (Figure 6(b) and Figure 6(c)) cannot explicitly explain why the $\mathrm{K}$ system exhibits, in experiments, better diffusion properties than the $\mathrm{Na}$ system. In fact, this places more emphasis on the role of activation energies (Figure 4(b)) and hence their diffusion coefficients, as well as the difference in the bottleneck radius (Figure 8) of the two systems, in order to ascertain this trend in experiment.

For a deeper understanding of the role of the interlayer distance in cationic diffusion at an atomistic level, the bottleneck radius formed by the oxygen atoms, was calculated along the cationic diffusion pathway. As defined by Evstigneeva and co-workers,[21] the bottleneck

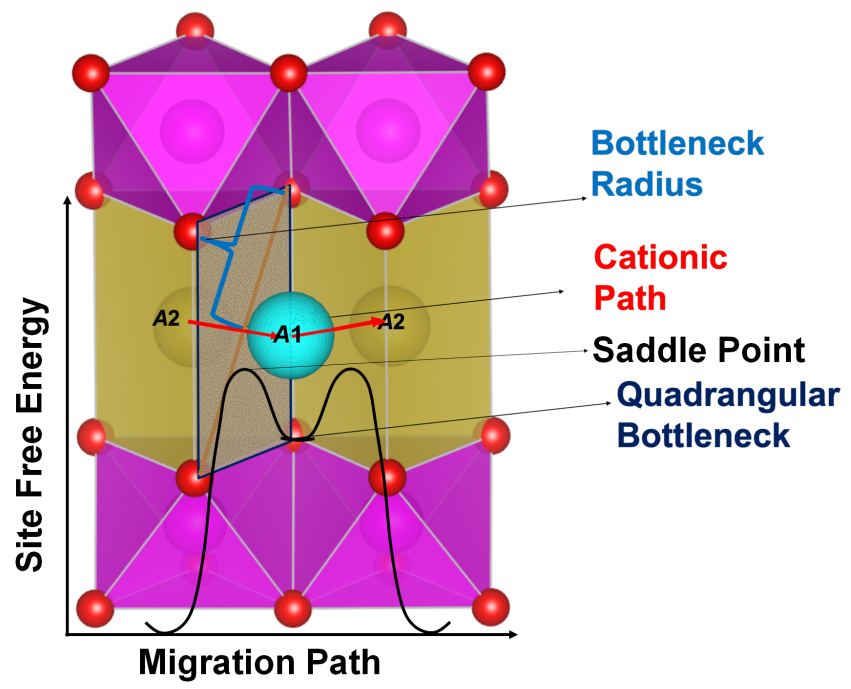

FIG. 8. Schematic representation of the cation-hopping path from $A 2$ to $A 1$ sites across the $A_{2} \mathrm{Ni}_{2} \mathrm{TeO}_{6}$ topology and the associated (relative) free energy landscape. The bottleneck radius and the cationic migration path have been highlighted. A curve illustrating the free-energy landscape is also appended, for clarity. The quadrangular bottleneck is located near the transition state (one of the bottleneck radii is shaded for emphasis). Different colours have been used for atoms residing in $A 1$ and $A 2$ sites, for clarity.

radius is the smallest $A-\mathrm{O}$ distance at the saddle point in the cationic diffusion path from the $A 1$ to $A 2$ sites, as shown in Figure 8. In the present study, the other bottlenecks formed between the path $A 1$ to $A 3$ are not taken into account, as they do not play any role on cationic diffusion owing to the cationic absence at the $A 3$-sites. The results indicate that the bottleneck distance for the $\mathrm{Na}$ system $(2.35 \AA)$ is smaller than the $\mathrm{K}$ system $(2.57 \AA)$ as is evident in Figure 9 . Thus, the $\mathrm{Na}$ system embodies a stronger force of attraction at the saddle points of the (relative) free energy path owing to the closer $\mathrm{O}$ atoms, effectively inhibiting cationic diffusion along the channels. Conversely, the $\mathrm{K}$ system manifests the weakest force of attraction at the saddle points due to the distant $\mathrm{O}$ atoms, yielding the highest cationic diffusion rate as observed in the MD simulation results. Figure 9 indeed depicts a similar trend. That is, larger inter-layer distances confer wider bottleneck radii; thus, resulting in higher cationic diffusion, as further displayed in Figure 5. By varying ionic radius whilst keeping the inter-layer distance fixed to $6.23 \AA$, corresponding to the value reported in measurements of the unpressurised $\mathrm{K}_{2} \mathrm{Ni}_{2} \mathrm{TeO}_{6}$, the effective bottleneck radius increases the smaller the cationic size. In other words, for a fixed bottleneck radius, a smaller-sized cation can easily pass through (compared to a larger-sized cation), thus resulting in higher cationic diffusion (as is evident in Figure S7). This behaviour is known as 'levitation effect', as studied elsewhere.[49] However, the diffusion 


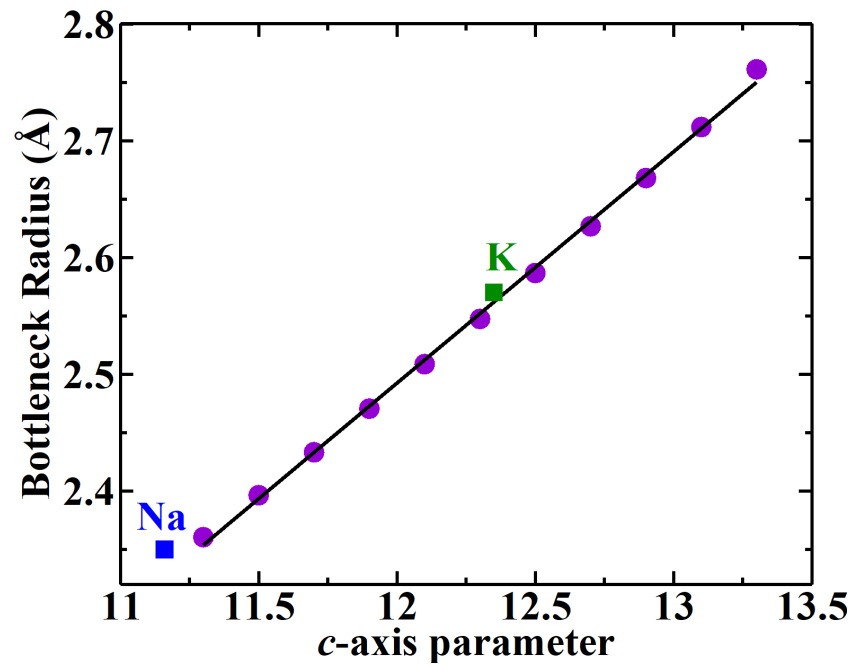

FIG. 9. Bottleneck radius formed by oxygen atoms located between the cationic path from $A 1$ to $A 2$ for the different $c$-axis parameters. The bottleneck radii for $\mathrm{Na}$ and $\mathrm{K}$ are marked using blue and green square symbols, respectively.

behaviour does not change as significantly as in the case of the change in inter-layer distance. Therefore, this clearly indicates the domination of inter-layer distance over the cationic size in establishing the leading order trends in the diffusion behaviour. The accurate depiction of the progressive increase in the bottleneck radius with increasing inter-layer distances reaffirms the use of MD simulations as an auspicious tool to decipher the atomistic mechanisms of honeycomb layered oxides.

In light of the observations presented in this study, it can be deduced that the de novo design of chemical compositions of $A_{2} \mathrm{Ni}_{2} \mathrm{TeO}_{6}$ entailing lithophile atoms with larger ionic sizes than $\mathrm{K}$ such as $A=\mathrm{Rb}$ or $\mathrm{Cs}$ could be a propitious strategy for enhancing the cationic diffusion for honeycomb layered oxide family discussed herein. However, MD simulations suggest that such systems are relatively unstable, because of the extremely weak Van der Waal interactions between adjacent layers. Stabilisation of such new compositions may necessitate the utilisation of high-pressure synthesis. Moreover, computation has also aided us to understand the high K-ion diffusion experimentally reported in $\mathrm{K}_{2} \mathrm{Ni}_{2} \mathrm{TeO}_{6}$ using muon spin rotation and relaxation spectroscopy.[28] The high K-ion diffusion is explained from the potential energy barrier (weaker cation and inter-layer interaction), favourable path connectivity and wider bottleneck radius.

\section{CONCLUSION}

This study shows the efficacy of using the VashishtaRahman potential in reproducing structural and transport properties of honeycomb layered oxides based on nickel tellurates and this potential model can be leveraged to garner insights on related honeycomb layered oxide materials. Moreover, the ability to depict, with high fidelity, the atomic scale cationic diffusion aspects of honeycomb layered oxides, avails a new avenue to test the ion dynamics of various honeycomb layered oxides when subjected to various conditions (e.g. pressure) presently unreported and currently inaccessible via experiment.

The interplay between inter-layer distance and cationic size in the ionic diffusion of honeycomb layered oxides is unveiled - a wider inter-layer distance favours faster diffusion, whereas a larger cationic size does not necessarily favour faster diffusion. Based on the computational results, cationic diffusion is influenced more by the inter-layer distance than the size of the cation. Moreover, we note that, there is an exponential increase in the ionic diffusion with increasing inter-layer distance, which puts forward the possibility to test various theoretical models[43] relating to ionic diffusion in honeycomb layered oxides as well as stimulate new experimental avenues that decouple the effects of inter-layer distance from the ionic radius such as applying pressure or recreating an expansion scenario to test the predicted trends herein for negative pressure.

Therefore, the results reported herein not only affirm the remarkable performance of honeycomb layered oxides but also validate the use of computation techniques such as molecular dynamic simulations as a propitious path in the quest to design high-performance materials for future capacious batteries.

\section{ACKNOWLEDGEMENTS}

This work was supported by the TEPCO Memorial Foundation. We gratefully acknowledge the Center for Computational Materials Science of Institute for Materials Research, Tohoku University for permitting the use of MASAMUNE- IMR (MAterial science Supercomputing system for Advanced MUltiscale simulations toward NExt-generation Institute of Material Research) (project no. 20S0021). This work was also conducted in part under the auspices of the Japan Society for the Promotion of Science (JSPS KAKENHI Grant Number 21K14730) and the National Institute of Advanced Industrial Science and Technology.
[1] A. Manthiram, Nature communications 11, 1 (2020).

[2] N. Nitta, F. Wu, J. T. Lee, and G. Yushin, Materials today $\mathbf{1 8}, 252(2015)$.
[3] M. Armand and J.-M. Tarascon, nature 451, 652 (2008).

[4] T. Hosaka, K. Kubota, A. S. Hameed, and S. Komaba, Chemical reviews 120, 6358 (2020). 
[5] N. Yabuuchi, K. Kubota, M. Dahbi, and S. Komaba, Chemical reviews 114, 11636 (2014).

[6] Y. Hironaka, K. Kubota, and S. Komaba, Chemical Communications 53, 3693 (2017).

[7] G. M. Kanyolo, T. Masese, N. Matsubara, C.-Y. Chen, J. Rizell, Z.-D. Huang, Y. Sassa, M. Månsson, H. Senoh, and H. Matsumoto, Chemical Society Reviews 50, 3990 (2021).

[8] A. J. Brown, Q. Xia, M. Avdeev, B. J. Kennedy, and C. D. Ling, Inorganic chemistry 58, 13881 (2019).

[9] J. Ma, S.-H. Bo, L. Wu, Y. Zhu, C. P. Grey, and P. G. Khalifah, Chemistry of Materials 27, 2387 (2015).

[10] A. Gupta, C. B. Mullins, and J. B. Goodenough, Journal of power sources 243, 817 (2013).

[11] L. Zheng and M. Obrovac, Journal of The Electrochemical Society 163, A2362 (2016).

[12] N. S. Grundish, I. D. Seymour, G. Henkelman, and J. B. Goodenough, Chemistry of Materials 31, 9379 (2019).

[13] Z. Yang, Y. Jiang, L. Deng, T. Wang, S. Chen, and Y. Huang, Journal of Power Sources 360, 319 (2017).

[14] D. Yuan, X. Liang, L. Wu, Y. Cao, X. Ai, J. Feng, and H. Yang, Advanced materials 26, 6301 (2014).

[15] T. Masese, K. Yoshii, Y. Yamaguchi, T. Okumura, Z.-D. Huang, M. Kato, K. Kubota, J. Furutani, Y. Orikasa, H. Senoh, et al., Nature communications 9, 1 (2018).

[16] T. Masese, K. Yoshii, M. Kato, K. Kubota, Z.-D. Huang, H. Senoh, and M. Shikano, Chemical Communications 55, 985 (2019).

[17] K. Yoshii, T. Masese, M. Kato, K. Kubota, H. Senoh, and M. Shikano, ChemElectroChem 6, 3901 (2019).

[18] C.-Y. Chen, J. Rizell, G. M. Kanyolo, T. Masese, Y. Sassa, M. Månsson, K. Kubota, K. Matsumoto, R. Hagiwara, and Q. Xu, Chemical Communications 56, 9272 (2020).

[19] D. S. Bhange, G. Ali, D.-H. Kim, D. A. Anang, T. J. Shin, M.-G. Kim, Y.-M. Kang, K. Y. Chung, and K.-W. Nam, Journal of Materials Chemistry A 5, 1300 (2017).

[20] H. Huang, Y. Yang, C. Chi, H.-H. Wu, and B. Huang, Journal of Materials Chemistry A 8, 22816 (2020).

[21] M. A. Evstigneeva, V. B. Nalbandyan, A. A. Petrenko, B. S. Medvedev, and A. A. Kataev, Chemistry of Materials 23, 1174 (2011).

[22] R. Berthelot, W. Schmidt, A. Sleight, and M. Subramanian, Journal of Solid State Chemistry 196, 225 (2012).

[23] D. K. Yadav, A. Sethi, S. Uma, et al., Dalton Transactions 48, 8955 (2019).

[24] V. Nalbandyan, A. Petrenko, and M. Evstigneeva, Solid State Ionics 233, 7 (2013).

[25] W. Schmidt, R. Berthelot, L. Etienne, A. Wattiaux, and M. A. Subramanian, Materials Research Bulletin 50, 292 (2014).

[26] V. Kumar, N. Bhardwaj, N. Tomar, V. Thakral, and S. Uma, Inorganic chemistry 51, 10471 (2012).

[27] S. K. Karna, Y. Zhao, R. Sankar, M. Avdeev, P. Tseng, C. Wang, G. Shu, K. Matan, G. Guo, and F. Chou, Physical Review B 95, 104408 (2017).
[28] N. Matsubara, E. Nocerino, O. K. Forslund, A. Zubayer, K. Papadopoulos, D. Andreica, J. Sugiyama, R. Palm, Z. Guguchia, S. P. Cottrell, et al., Scientific reports 10, 1 (2020).

[29] A. K. Bera and S. M. Yusuf, The Journal of Physical Chemistry C 124, 4421 (2020).

[30] P. P. Kumar and S. Yashonath, Journal of Chemical Sciences 118, 135 (2006).

[31] K. Sau and P. P. Kumar, The Journal of Physical Chemistry C 119, 1651 (2015).

[32] K. Sau and P. P. Kumar, The Journal of Physical Chemistry C 119, 18030 (2015).

[33] K. Sau, Ionics 22, 2379 (2016).

[34] K. Sau, T. Ikeshoji, and S. Roy, Physical Chemistry Chemical Physics 22, 14471 (2020).

[35] P. Vashishta and A. Rahman, Physical Review Letters 40, 1337 (1978).

[36] R. A. Buckingham, Proceedings of the Royal Society of London. Series A. Mathematical and Physical Sciences 168, 264 (1938).

[37] J. E. Lennard-Jones, Proceedings of the Physical Society (1926-1948) 43, 461 (1931).

[38] P. P. Kumar and S. Yashonath, The Journal of Physical Chemistry B 106, 7081 (2002).

[39] K. Pramanik, K. Sau, and P. P. Kumar, The Journal of Physical Chemistry C 124, 4001 (2020).

[40] M. Parrinello and A. Rahman, Journal of Applied physics 52, 7182 (1981).

[41] S. Plimpton, Journal of computational physics 117, 1 (1995).

[42] E. Snider, N. Dasenbrock-Gammon, R. McBride, M. Debessai, H. Vindana, K. Vencatasamy, K. V. Lawler, A. Salamat, and R. P. Dias, Nature 586, 373 (2020).

[43] G. M. Kanyolo and T. Masese, Scientific reports 10, 1 (2020).

[44] For instance, for a perfect fluid of particles it is given by $P=w \rho,[50]$ where $w$ is a parameter characterizing the fluid; for instance, the ideal gas law formula $P V=$ $n R T$ corresponds to $\rho=n / V$ and $w=R T$, where $n$ is the atomic number of gas molecules and $R$ is the gas constant. In materials, the parameter $w$ can also depend on the radial distribution function, $g(r)[45]$.

[45] M. Tuckerman, Statistical mechanics: theory and molecular simulation (Oxford university press, 2010).

[46] B. Bhargava and S. Balasubramanian, The Journal of chemical physics 123, 144505 (2005).

[47] J. P. Hansen and I. R. McDonald, Physical Review A 11, 2111 (1975).

[48] F. Dommert, J. Schmidt, B. Qiao, Y. Zhao, C. Krekeler, L. Delle Site, R. Berger, and C. Holm, The Journal of chemical physics 129, 224501 (2008).

[49] P. Padma Kumar and S. Yashonath, The Journal of Physical Chemistry B 106, 3443 (2002).

[50] N. Mazumder and S. Chakraborty, Astrophysics and Space Science 330, 137 (2010). 